\title{
Influencia de la obesidad sobre la resistencia y conductancia específica de la vía aérea en niños escolares
}

\author{
The impact of obesity on specific airway resistance and \\ conductance among schoolchildren
}

\author{
Lic. Andrea Parraguez Arévalo ${ }^{a}$, Lic. Francisco Rojas Navarro ${ }^{a}$, \\ Lic. Macarena Ruz Céspedes ${ }^{a}$, MSc. Paul Medina González ${ }^{b}$, \\ MSc. Máximo Escobar Cabello y MSc. Rodrigo Muñoz Cofréc
}

\section{a. Escuela de Kinesiología, Universidad Católica del Maule, Chile. \\ b. Departamento de Kinesiología, Facultad de Ciencias de la Salud, Universidad Católica del Maule, Chile. \\ c. Programa de Doctorado en Ciencias Morfológicas, Universidad de La Frontera, Chile.}

Correspondencia: MSc. Rodrigo Muñoz Cofré: rodrigomunozcofre@ gmail.com

\section{Financiamiento:}

Ninguno.

Conflicto de intereses: Ninguno que declarar.

Recibido: 24-6-2017

Aceptado: 13-9-2017

\section{RESUMEN}

Introducción. La obesidad infantojuvenil es un problema epidemiológico en los países en vías de desarrollo. Su prevalencia entre los niños en edad preescolar y escolar supera el $30 \%$. Esta se asocia a una amplia variedad de complicaciones de salud, entre ellas, una acelerada pérdida de la función pulmonar, que provoca cambios en la fisiología y mecánica ventilatoria. El objetivo de este estudio fue estudiar la asociación entre obesidad y aumento de la resistencia específica de las vías aéreas (specific resistance airway; sRaw, por sus siglas en inglés) en una muestra infantojuvenil obesa de la comuna de Talca.

Material y método. En una muestra de 36 sujetos con un promedio de edad de 9,38 $\pm 1,99$ años, dividida en dos grupos, peso normal y obesos, se midieron los pliegues tricipital, subescapular, abdominal y volúmenes pulmonares. Para el análisis estadístico, se determinó la normalidad de los datos y, posteriormente, se utilizaron los test t deStudent oU de Mann-Whitney y Pearson o Spearman según correspondiera. Se consideró un nivel de significancia estadística de $p<0,05$. Resultados. Al comparar sujetos de peso normal con obesos, se observó un aumento significativo de la sRaw y una disminución significativa de la conductancia específica de la vía aérea en sujetos obesos. Además, se observó una correlación buena y significativa entre sRaw y porcentaje de grasa.

Conclusiones. Los sujetos obesos mostraron un aumento de la sRaw y disminución de la conductancia específica de la vía aérea.

Palabras clave: obesidad, resistencia de las vías respiratorias.

http:/ / dx.doi.org/10.5546/ aap.2018.e227

Texto completo en inglés:

http:/ / dx.doi.org/10.5546/ aap.2018.eng.e227

Cómo citar: Parraguez Arévalo A, Rojas Navarro F, Ruz Céspedes M, et al. Influencia de la obesidad sobre la resistencia y conductancia específica de la vía aérea en niños escolares. Arch Argent Pediatr 2018;116(2):e227-e233.

\section{INTRODUCCIÓN}

En la actualidad, el aumento en la tecnología ha disminuido los niveles de actividad física en el hombre, ${ }^{1}$ lo que impacta en su estilo de vida. Una de las consecuencias más críticas es la aparición de la obesidad, cuya extensión o prevalencia llega a involucrar, inclusive, a los individuos en su más temprana edad. ${ }^{2}$

En este contexto, la Organización Mundial de la Salud (OMS) define "obesidad" como “una acumulación anormal o excesiva de grasa que puede ser perjudicial para la salud'. Hoy en día, su prevalencia va en alza y un grupo de alto riesgo está representado por los niños obesos, debido a que un porcentaje de ellos mantendrán esta enfermedad en la edad adulta. ${ }^{3}$ Es un hecho no menor considerando que, en el año 2010, alrededor de 40 millones de niños menores de cinco años ya tenían sobrepeso. ${ }^{4}$ Nuestro país no escapa a esta realidad, pues sigue una curva ascendente desde el año 2009. No obstante, aún no son totalmente dimensionados los efectos en la función pulmonar.

La masa grasa característica de la obesidad es el componente de mayor variabilidad en la composición corporal a lo largo del ciclo vital, tanto en un grupo de sujetos como en cada persona. Así, los riesgos asociados al exceso de grasa se deben, en gran porcentaje, a su localización. ${ }^{4}$ De este modo, se postula que las consecuencias metabólicas de la distribución regional de la grasa 
tienen particular importancia clínica, debido a las disfunciones sistémicas que provocaría. ${ }^{5}$ Esta situación se verá potenciada por factores intrínsecos, como la etnia, el genotipo, la dieta, la actividad física, el género y la edad. ${ }^{6}$

La obesidad altera directamente las propiedades del tórax y los pulmones mediante la acumulación de grasa en el mediastino, el abdomen y la cavidad torácica. ${ }^{7}$ Los efectos mecánicos e inflamatorios de esta enfermedad provocan cambios en la fisiología ventilatoria. ${ }^{8,9}$ Se eleva el diafragma y provoca un aumento de la presión pleural y una disminución de la capacidad residual funcional. Paralelamente, el tejido adiposo produce citoquinas proinflamatorias y hormonas que afectan de manera directa las vías aéreas y disminuyen su calibre. ${ }^{10} \mathrm{Al}$ respecto, la extensión periférica de su compromiso en escolares sanos ha sido menos estudiada.

Asimismo, la distribución regional de la grasa juega un papel importante en la predisposición de los sujetos obesos a complicaciones respiratorias. ${ }^{11,12}$ En este contexto, se ha observado en niños obesos que existe una correlación inversa entre la capacidad vital forzada (CVF) y el índice de masa corporal (IMC). ${ }^{10}$ Complementariamente, Shenoy et al. encontraron una correlación directa entre la distribución de grasa abdominal y el volumen espiratorio forzado en el primer segundo $\left(\mathrm{VEF}_{1}\right){ }^{13}$ Por el contrario, en niños de peso normal, se ha demostrado una relación directa e inversa entre el IMC y la resistencia de las vías aéreas medida por oscilometría de impulso. ${ }^{14}$ Sin embargo, estas investigaciones son espirométricas y no miden la resistencia específica de las vías aéreas (specific resistance airway; sRaw, por sus siglas en inglés) ni la conductancia específica de la vía aérea (specific conductance airway; sGaw, por sus siglas en inglés).

Considerando los antecedentes presentados, el objetivo del siguiente trabajo es estudiar la asociación entre obesidad y aumento de la resistencia de la vía aérea en una muestra infantojuvenil obesa de la comuna de Talca.

\section{MATERIAL Y MÉTODO}

Investigación observacional de corte transversal, que se realizó entre los meses de marzo y mayo del presente 2017. El tamaño de la muestra se calculó utilizando el software Cálculo de tamaño muestral Ene 3, $0^{\circledR}$. Se determinó un nivel de significancia de 0,95 , una potencia estadística de $80 \%$, un número de dos grupos, un porcentaje de abandono de $10 \%$ y una media y desviación estándar determinada desde un estudio piloto, que arrojó un total de 36 sujetos. A través de muestreo no probabilístico, se seleccionaron estos 36 individuos, todos de la ciudad de Talca, quienes, de acuerdo con su IMC (percentiles de la OMS), se dividieron en dos grupos: 18 de peso normal y 18 obesos. Los criterios de inclusión fueron niños de la comuna de Talca, de entre 6 y 12 años, de peso normal (IMC para la edad dentro de desviación típica establecida por la mediana en los patrones de crecimiento infantil de la OMS) y obesos (IMC para la edad con más de una desviación típica por encima de la mediana establecida en los patrones de crecimiento infantil de la OMS). ${ }^{15}$ Los criterios de exclusión fueron incompatibilidad del estado físico y / o cognitivo para realizar la prueba de función pulmonar, enfermedades respiratorias agudas o crónicas al momento de la evaluación, niñas que hubieran presentado menarquia, náuseas y vómitos el día del examen. ${ }^{10,16}$ Este estudio contó con la aprobación del Comité de Ética Científica de la Universidad Católica del Maule (Acta de Resolución 23/2016). Por último, los padres de los escolares debieron firmar el consentimiento informado y, antes de cada evaluación, se le solicitó el asentimiento a cada escolar.

Estatura: Se midió con un antropómetro SECA $^{\circledR} 220$ la distancia desde el suelo al vertex. El sujeto debió estar de pie, con los talones juntos y los pies formando un ángulo de $45^{\circ}$. La medida fue en inspiración máxima, manteniendo la cabeza en el plano de Frankfort. ${ }^{17}$

Peso: Se midió con una balanza SECA ${ }^{\circledR} 840$ el peso en kilogramos. Importante: el sujeto no debía ver su registro. ${ }^{17}$

Pliegues: Con un caliper Lange Skinfold Caliper ${ }^{\circledR}$ (Beta Technology, Santa Cruz, California), se midió el tejido adiposo expresado en milímetros. Los pliegues fueron los siguientes: tricipital, que era vertical y se situaba en el punto medio acromiorradial de la cara posterior del brazo; subescapular, que estaba ubicado a dos centímetros del ángulo inferior de la escápula, en dirección oblicua hacia abajo y afuera formando un ángulo de $45^{\circ}$ con la horizontal; abdominal, a cinco centímetros a la derecha del ombligo, se tomó de manera longitudinal a la línea media del cuerpo. ${ }^{17}$

Porcentaje de grasa: Se determinó a través de la ecuación de Slaughter para pre-púberes. ${ }^{17,18}$

Mujeres: 1,33 (tricipital + subescapular) - 0,013 $(\text { tricipital }+ \text { subescapular })^{2}-2,5$. 
Hombres: 1,21 (tricipital + subescapular) $0,008$ (tricipital + subescapular) $)^{2}-1,7$.

Volúmenes pulmonares: Las pruebas se realizaron en un pletismógrafo corporal (Medgraphics Modelo PlatinumElite DL ${ }^{\circledR}$ ). De manera breve, se ajustó el neumotacógrafo a la altura de la boca. El evaluado se ubicó el clip nasal y las manos en la musculatura facial para bloquear su utilización en la prueba. Posteriormente, se cerró la cabina y se le indicó realizar cuatro ventilaciones a volumen corriente. Se instruyó al sujeto para que "jadeara suavemente" intentando mover volúmenes de entre 50 y $60 \mathrm{ml}$ y a una frecuencia cercana a 60 por minuto $(1 \mathrm{~Hz})$. Luego se activó el shutter durante 2-3 segundos y, después de esto, se indicó una inspiración máxima y una espiración máxima hasta volumen residual. ${ }^{16}$

Los datos se tabularon y analizaron en los programas Microsoft Office Excel 2010 y GraphPad Prism $5^{\circledR}$, respectivamente. Los resultados se presentaron como promedios \pm 1 desviación estándar. Para el análisis estadístico de las variables sRaw y sGaw, se determinó la normalidad de los datos a través de la prueba de Shapiro-Wilk; posteriormente, se usó $t$ de Student o U de Mann-Whitney dependiendo de la distribución de la muestra. Para las correlaciones, se utilizó el coeficiente de Pearson o Spearman, dependiendo de la normalidad de los datos. Por último, se consideró un nivel de significancia de $p<0,05$.

\section{RESULTADOS}

Se evaluaron 36 niños; no existió diferencia significativa en edad y talla (Tabla 1 ). El análisis estadístico también mostró diferencias estadísticamente significativas en las variables de peso e IMC (Tabla 1). Los pliegues abdominal $\mathrm{y}$ tricipital fueron significativamente mayores en niños obesos que en los de peso normal (Tabla 1). Para la variable de sRaw, se encontró un aumento significativo en obesos en relación con los niños de peso normal, con una $p=0,0001$. La sGaw disminuyó significativamente en niños obesos con respecto a los de peso normal, con una $p=0,0004$ (Figura 1).

Además, se observó una correlación buena y significativa entre sRaw y porcentaje de grasa (Figura 2). No se observaron correlaciones estadísticamente significativas entre sRaw y los pliegues subescapular y abdominal (Figuras 3 y 4). Por último, no se encontraron correlaciones estadísticamente significativas entre sGaw y porcentaje de grasa, pliegue subescapular y abdominal (Figura 5).

\section{DISCUSIÓN}

La precocidad con que se puedan detectar los efectos sistémicos de la obesidad y la distribución

TABLA 1. Descripción de variables antropométricas y de función ventilatoria de la población infantojuvenil evaluada de la comuna de Talca

\begin{tabular}{|c|c|c|c|}
\hline Variables & Peso normal & Obeso & Valor de $p$ \\
\hline Muestra total $(\%)$ & $18(50)$ & $18(50)$ & - \\
\hline Sexo $(H-M)$ & $14 / 4$ & $7 / 11$ & - \\
\hline Edad (años) & $9,88 \pm 1,60$ & $8,88 \pm 2,08$ & $0,1789^{\S}$ \\
\hline Peso (kg) & $34,36 \pm 6,39$ & $49,21 \pm 12,61$ & $0,0002^{\ddagger}$ \\
\hline Talla $(\mathrm{m})$ & $1,41 \pm 0,11$ & $1,38 \pm 0,11$ & $0,4063^{\ddagger}$ \\
\hline $\operatorname{IMC}\left(\mathrm{kg} / \mathrm{m}^{2}\right)$ & $17,12 \pm 1,17$ & $25,50 \pm 3,26$ & $0,0001^{\ddagger}$ \\
\hline Predicho & $16,81 \pm 0,75$ & $16,46 \pm 0,97$ & - \\
\hline$\%$ del predicho & $101,93 \pm 6,40$ & $154,97 \pm 18,67$ & $0,0001^{\ddagger}$ \\
\hline P. subescapular (mm) & $6,99 \pm 2,57$ & $20,57 \pm 6,75$ & $0,0001^{\S}$ \\
\hline P. abdominal (mm) & $12,70 \pm 6,56$ & $33,45 \pm 9,29$ & $0,0001^{\ddagger}$ \\
\hline Grasa corporal (\%) & $17,63 \pm 5,21$ & $31,68 \pm 4,68$ & $0,0001^{\ddagger}$ \\
\hline sRaw $\left(\mathrm{cmH}_{2} \mathrm{O} \cdot \mathrm{s}^{-1}\right)$ & $3,60 \pm 0,94$ & $5,33 \pm 1,03$ & $0,0001^{\ddagger}$ \\
\hline Predicho & $<4,76$ & $<4,76$ & - \\
\hline$\%$ del predicho & - & - & - \\
\hline $\operatorname{sGaw}\left(1 / \mathrm{cmH}_{2} \mathrm{O}^{-1} \mathrm{~s}^{-1}\right)$ & $0,30 \pm 0,0$ & $0,20 \pm 0,04$ & $0,0004^{\ddagger}$ \\
\hline Predicho & 0,19 & 0,19 & - \\
\hline$\%$ del predicho & $156,39 \pm 45,17$ & $95,61 \pm 30,15$ & $0,0001^{\ddagger}$ \\
\hline
\end{tabular}

H: hombre; M: mujer; kg: kilogramos; m: metros; kg/m²: kilogramos partidos por metros al cuadrado; P.: pliegue; mm: milímetros; sRaw: resistencia específica de la vía aérea; sGaw: conductancia específica de la vía aérea; $\mathrm{cmH}_{2} \mathrm{O}^{-1}{ }^{-1}$ : centímetros de agua por segundos; §: U de Mann-Whitney; ¥: $\mathrm{t}$ de Student. 
de la grasa corporal es relevante para todo sujeto en desarrollo, sobre todo, cuando esta involucra funciones vitales, como la ventilación.

Los resultados del presente estudio indican que, en los niños obesos de la muestra, se puede asociar un aumento de su sRaw y una disminución de la sGaw. Sin embargo, una de las dificultades de estimar obesidad por el IMC es que no distingue entre grasa y masa corporal magra. ${ }^{19}$ En este sentido, Pérez et al. señalan que, dentro de las herramientas antropométricas específicas para la medición de grasa corporal, la circunferencia de cadera y los pliegues son instrumentos sencillos y rápidos de aplicar en la evaluación clínica y que, además, complementarían el resultado del IMC al observar la existencia de tejido graso y su ubicación. Pese a que el porcentaje de masa grasa obtenido desde los pliegues cutáneos tiene un nivel de concordancia bajo (según Bland y Altman), entre distintos evaluadores, ${ }^{20,21}$ Urrejola et al. sugieren utilizar este método mientras no se desarrollen herramientas de mayor confiabilidad..$^{22} \mathrm{Un}$ aspecto relacionado con las consecuencias de la obesidad sobre la función ventilatoria, pero a la vez poco estudiado, es el aumento de la sRaw y el descenso de la sGaw, ${ }^{23}$ debido a que, por lo general, son utilizadas como pruebas específicas para patologías de la

FiguRA 1. Comparación de la resistencia y conductancia específica de las vías aéreas según el índice de masa corporal de la población infantojuvenil evaluada de la comuna de Talca

A.

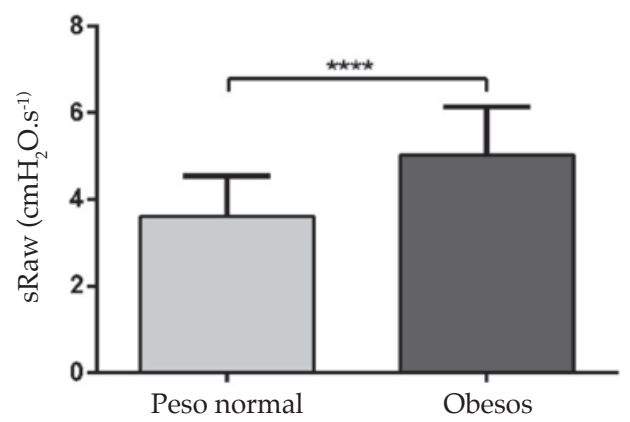

B.

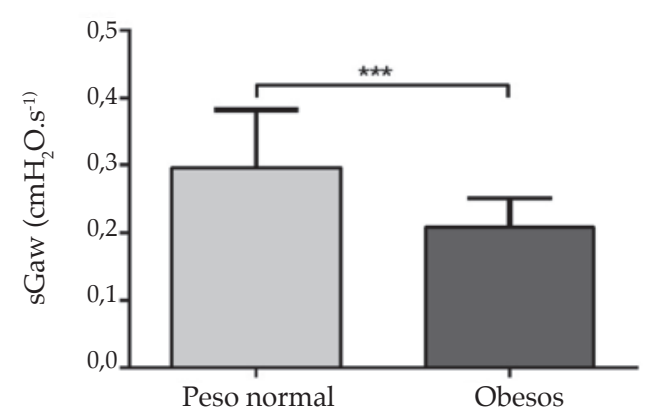

$\mathrm{cmH}_{2} \mathrm{O} \cdot \mathrm{s}^{-1}$ : centímetros de agua por segundos; ${ }^{* * *} p<0,0001 ;{ }^{* * *} p=0,0004$.

A. Comparación de la sRaw entre los grupos de peso normal y obesos. B. Comparación de la sGaw entre peso normal y obesos. SRaw: resistencia específica de la vía aérea; sGaw: conductancia específica de la vía aérea.

FiguRA 2. Relación del porcentaje de grasa y resistencia específica de las vías aéreas en la población infantojuvenil obesa de la comuna de Talca

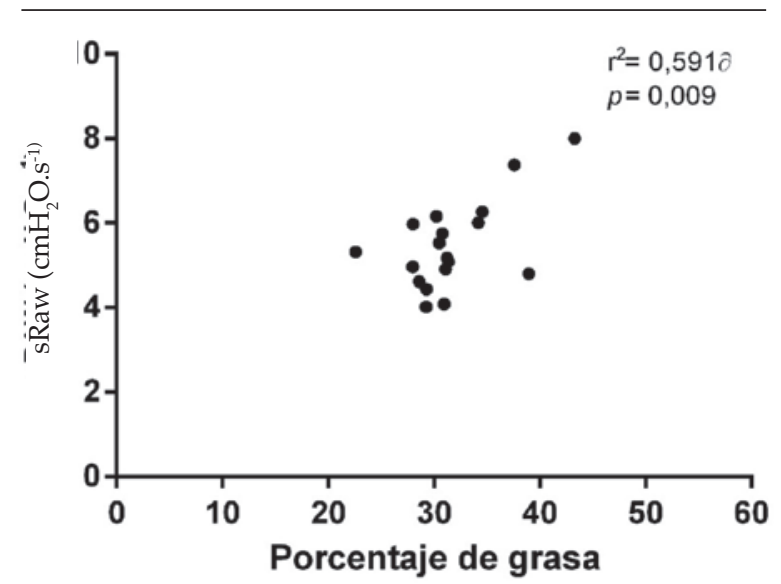

sRaw: resistencia específica de la vía aérea; $\mathrm{cmH}_{2} \mathrm{O} . \mathrm{s}^{-1}$ : centímetros de agua por segundos; $\mathrm{r}^{2}$ : Pearson.
FIGURA 3. Relación del pliegue subescapular y la resistencia específica de las vías aéreas en la población infantojuvenil obesa de la comuna de Talca

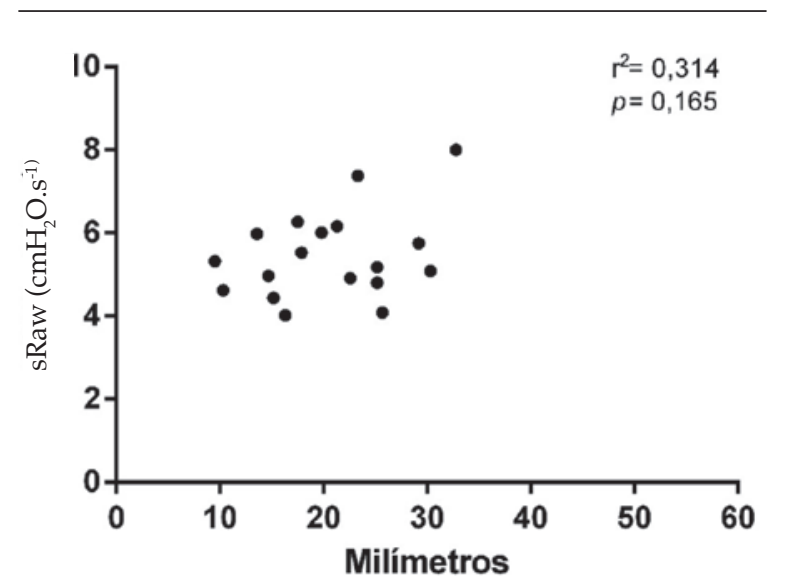

sRaw: resistencia específica de la vía aérea; $\mathrm{cmH}_{2} \mathrm{O} \cdot \mathrm{s}^{-1}$ : centímetros de agua por segundos. 
vía aérea ${ }^{24}$ y a que no necesariamente guardan relación con la composición corporal del sujeto. A partir de la información obtenida, por lo menos, se podría considerar la evaluación de la función pulmonar en sujetos obesos que aparentemente no presentaran trastornos respiratorios.

En detalle, la sRaw de los grupos evaluados (Figura 1) se comportó de manera distinta a estudios anteriores. Al respecto, Kalhoff et al. estudiaron la asociación entre los parámetros oscilométricos y el IMC en un grupo de 518 preescolares (10\% con sobrepeso y $3 \%$ de obesos), y los resultados no mostraron cambios significativos en la resistencia de las vías aéreas con el incremento del IMC. Los autores atribuyeron estos resultados a la homogeneidad

FIGURA 4. Relación del pliegue abdominal y la resistencia específica de las vías aéreas en la población infantojuvenil obesa de la comuna de Talca

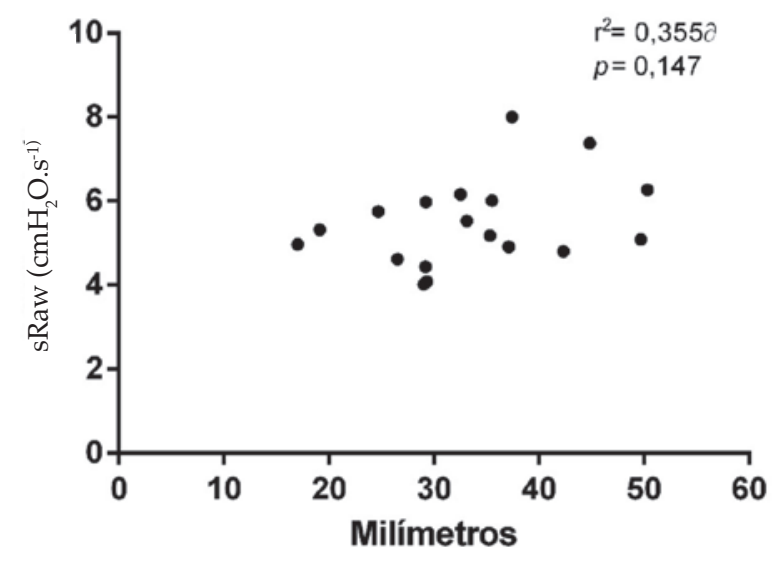

sRaw: resistencia específica de la vía aérea; $\mathrm{cmH}_{2} \mathrm{O} . \mathrm{s}^{-1}$ : centímetros de agua por segundos; $\mathrm{r}^{2}$ : Pearson. de la muestra y también a que los sujetos evaluados eran sanos. ${ }^{14}$ Del mismo modo, Lauhkonen et al. evaluaron la asociación entre el peso, la función pulmonar y la reactividad bronquial con el ejercicio en preescolares a través de la oscilometría de impulso. Para esto, midieron a 99 niños (22\% de obesos), los cuales fueron evaluados después de una bronquiolitis (0-6 meses) y reevaluados a los 6 años. Los niños obesos, en comparación con los de peso normal y con sobrepeso, no demostraron diferencias significativas en resistencia e impedancia de las vías aéreas, tanto basal como posejercicio. Sin embargo, siete niños obesos mostraron una mayor impedancia y menor resistencia de las vías aéreas posbroncodilatador. Los investigadores, a partir de la evidencia preliminar, demostraron que la obesidad actual (5-7 años) en niños hospitalizados por bronquiolitis entre 0 y 6 meses podía estar asociada con la obstrucción bronquial, que incluía la obstrucción irreversible después de la administración de broncodilatadores. Además de esto, no se encontró asociación significativa entre sobrepeso u obesidad actual y la reactividad de las vías aéreas en el ejercicio y en la prueba de broncodilatación. ${ }^{25}$ Las diferencias reportadas por los dos artículos desarrollados anteriormente en relación con nuestro estudio se deberían a la edad de nuestros sujetos de estudios, entre 6 y 12 años, lo cual permitió mayor cooperación, al equilibrio en el número de niños en ambos grupos y al instrumento que se utilizó para realizar las mediciones.

Considerando que la American Thoracic Society (ATS) define la sGaw como una variable recíproca a la sRaw, la cual disminuye de manera lineal al caer el volumen pulmonar y aumentar la sRaw, este concepto es consistente con los resultados del

FIGURA 5. Influencia del porcentaje de grasa, pliegue subescapular y abdominal en la conductancia especifica de las vías aéreas de la población infantojuvenil obesa de la comuna de Talca
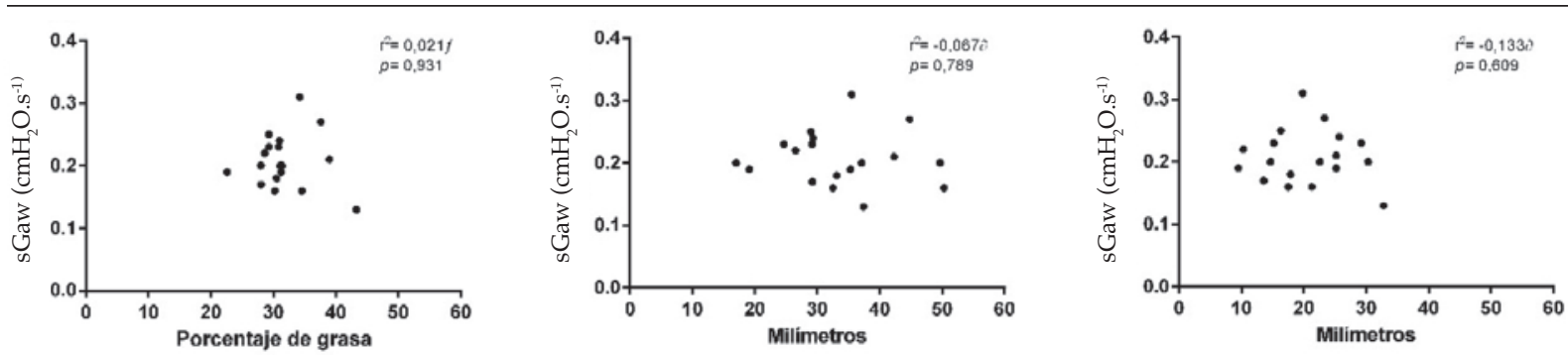

sGaw: conductancia específica de la vía aérea; $1 / \mathrm{cmH}_{2} \mathrm{O}_{\text {s }} \mathrm{s}^{-1}$ : uno partido por centímetros de agua por segundos; $f$ : Spearman; $\mathrm{r}^{2}$ : Pearson. Detalle de la conductancia específica de la vía aérea en el grupo de obesos.

A. Relacionado con el porcentaje de grasa. B. Relacionado con el pliegue subescapular. C. Relacionado con el pliegue abdominal. 
estudio, en el que se encontró una disminución significativa de la sGaw en los niños obesos. ${ }^{13}$ Por tanto, la categorización antropométrica según el IMC, sin ser una herramienta de alta complejidad, entregaría resultados que permitirían especular posibles trastornos del sistema ventilatorio de niños obesos y recomendar una evaluación de la función pulmonar.

En relación con la distribución de grasa corporal, Rosales señaló que existía información controversial sobre su comportamiento en niños y adolescentes. Al mismo tiempo, se desconoce con precisión sobre su depósito, ya sea central (tronco) o periférico (miembros). ${ }^{26}$ En este aspecto, Godínez et al., en su revisión sobre grasa abdominal e importancia en la obesidad, concluyen que su distribución en niños es en la zona central (tórax y abdomen), hecho que le da soporte a las evaluaciones utilizadas. ${ }^{27}$

A pesar de utilizar los pliegues cutáneos con el objetivo de calcular el porcentaje de grasa corporal, según la ecuación de Slaughter, también se registró la correlación de los pliegues subescapular y abdominal con la sRaw y sGaw. Sin embargo, esta no dio resultados significativos, tal vez, porque los pliegues por sí solos no daban cuenta de este fenómeno, a diferencia del porcentaje de grasa, que era una estimación indirecta de la totalidad de la grasa corporal (Tabla 1). Esto concordó con los resultados obtenidos por Frainer et al., quienes observaron, en niños brasileños de 7 a 10 años de edad, un patrón de distribución de grasa abdominal. ${ }^{28}$ Asimismo, McCarthy et al. midieron por bioimpedancia a niños y adolescentes de 5 a 18 años: obtuvieron un porcentaje de grasa corporal similar por sexo y determinaron que existía una ubicación abdominal de esta hasta los 10 años. ${ }^{29}$ La evidencia disponible sugiere que no existen diferencias por sexo en los patrones de distribución de grasa corporal en el rango etario evaluado, por lo que la separación por género en esta etapa del ciclo vital no sería necesaria.

Por último, es importante destacar la correlación significativa entre el porcentaje de grasa corporal y la sRaw. Esta concuerda con los resultados obtenidos por Feng et al., quienes, al medir una muestra de 1572 sujetos de 9 a 18 años, encontraron correlaciones inversas entre la distribución de grasa corporal obtenida a través de la circunferencia de cadera y tórax, con $\mathrm{VEF}_{1}$ y flujo espiratorio máximo (FEM). ${ }^{11}$ Si bien nuestro estudio midió la sRaw y no las variables antes mencionadas, cabe destacar que todas son traductoras de aumento en la resistencia de las vías aéreas, aunque la sRaw es altamente específica y sensible a trastornos obstructivos en comparación con el $\mathrm{VEF}_{1}$ y el FEM. Se cree que la explicación a este aumento en la sRaw descansa sobre dos hipótesis. La primera indica que el músculo liso es infiltrado por tejido graso, lo que provoca un aumento de la presión crítica de las vías respiratorias y favorece su colapso y anomalías estructurales del sistema ventilatorio. ${ }^{28}$ La segunda se sustenta en un incremento del estado inflamatorio crónico en las vías aéreas secundario a la obesidad. Aquí, la leptina (hormona satélite) generaría un ambiente proinflamatorio, el cual disminuye el radio de las vías aéreas a través de un aumento en el tono colinérgico e hiperreactividad persistente de las estas. ${ }^{8}$ Por ello, se podría pensar que este estado proinflamatorio continuo derivado del porcentaje de grasa total, el cual es mayor en obesos que con peso normal, estaría provocando la correlación entre sRaw y porcentaje de grasa, y no entre los pliegues subescapular y abdominal por separado. Esto es respaldado por Peters et al., quienes reportaron una directa relación entre leptina e hiperreactividad de las vías aéreas. ${ }^{8}$

\section{LIMITACIONES}

Las bajas temperaturas y la utilización de leña para la calefacción que se observa desde mayo en adelante generan un aumento del material particulado ${ }^{2,5,30}$ que podría generar inflamación de la vía aérea. Sería recomendable, para futuras experiencias, despejar este factor $\mathrm{y}$, así, aislar de mejor manera el factor obesidad. Además, debido a la discrepancia existente respecto a la distribución corporal de grasa en niños, habría sido conveniente haber usado ecuaciones con un mayor número de pliegues. Por último, el tamaño muestral fue calculado en relación con el objetivo del estudio y no para comprobar si las sustancias inflamatorias propias de la obesidad provocaban esta disminución en la sRaw.

\section{CONCLUSIÓN}

Existe una vinculación entre obesidad y función ventilatoria, y se observa un aumento significativo de la sRaw y una disminución significativa de la sGaw. Además, se mantiene una relación buena y significativa entre sRaw y el porcentaje de grasa de población infantojuvenil obesa de 6 a 12 años de edad de la ciudad de Talca. 


\section{REFERENCIAS}

1. Chakravarthy M, Booth F. Eating, exercise, and "thrifty" genotypes: Connecting the dots toward an evolutionary understanding of modern chronic diseases. J Appl Physiol (1985) 2004;96(1):3-10.

2. Ferrer Arrocha M, Rodríguez Fernández C, González Pedroso M, et al. Obesidad, hipertensión y tabaquismo: señales ateroscleróticas tempranas en adolescentes de la secundaria básica "Guido Fuentes". Rev Cubana Invest Biomed 2009;28(2): 20-30.

3. Soca P, Niño Peña A. Consecuencia dela obesidad. ACIMED. 2009;20(4):84-92.

4. Organización Mundial de la Salud. Sobrepeso y obesidad infantiles. 2013. [Acceso: 9 de octubre de 2016]. Disponible en: http: / / www.who.int/dietphysicalactivity / childhood/es/.

5. Quiles Molina M, Santos Ramírez B, Córdova Mendoza $\mathrm{C}$, et al. Correlación entre la función respiratoria y la obesidad en niños, en niños de 10 a 12 años de edad. Salud Tab 2007;13(1):568-72.

6. Shuster A, Patlas M, Pinthus J, et al. The clinical importance of visceral adiposity: a critical review of methods for visceral adipose tissue analysis. Br J Radiol 2012;85(1009):1-10.

7. Xanthopoulos M, Tapia IE. Obesity and common respiratory diseases in children. Paediatr Respir Rev 2017;23:68-71.

8. Peters U, Suratt BT, Bates JHT, et al. Beyond BMI: Obesity and Lung Diseases. Chest. 2017 [En prensa].

9. Muiño A, Torello P, Brea S. Función pulmonar en niños asmáticos con sobrepeso-obesidad. Arch Pediatr Urug 2009;80(2):109-15.

10. Brito Portuondo A, Hernández Perera J, Sánchez López L. Efectos de la obesidad sobre la función cardiovascular, respiratoria e inflamatoria. Invest Medicoquir 2012;4(2): 227-33.

11. Feng K, Chen L, Han S, et al. Ratio of waist circumference to chest circumference is inversely associated with lung function in Chinese children and adolescents. Respirology 2012;17(7):1114-8.

12. Chen Y, Rennie D, Cormier Y, et al. Waist Circumference Associated With Pulmonary Function in Children. Pediatr Pulmonol 2009;44(3):216-21.

13. Porhomayon J, Papadakos P, Sinhg A, et al. Alteration in respiratory physiology in obesity for anesthesia-critical care physician. HSR Proc Intensive Care Cardiovasc Anesth 2011;3(2):109-18.

14. Kalhoff $\mathrm{H}$, Breidenbach $\mathrm{R}$, Smith $\mathrm{H}$, et al. Impulse oscillometry in preschool children and association with body mass index. Respirology 2011;16(1):174-9.

15. WHO. Growth reference 5-19 years. [Acceso: 23 de agosto de 2017]. Disponible en http: / / www.who.int/growthref/ who2007_bmi_for_age/en/.
16. Jiang G, Li A, Wang L, et al. Reference data for BabyBodyplethysmographic measurements in Chinese neonates and infants. Respirology 2017. [En prensa].

17. International Society for the Advancement of Kinanthropometry. International Standards for Anthroponetric Assessement. Underdale: ISAK; 2001. [Acceso: 22 de agosto de 2017]. Disponible en http: / / www. ceap.br/material/MAT17032011184632.pdf.

18. Gómez Campo R, De Marco A, De Arruda M, et al. Predicción de ecuaciones para el porcentaje de grasa a partir de circunferencias corporales en niños pre-púberes. Nutr Hosp 2013;28(3):772-8.

19. Liyanage G, Jayamanne B D, Aaqiff M, et al. Effect of body mass index on pulmonary function in children. Ceylon Med J 2016;61(4):163-6.

20. Martínez E. Composición corporal: su importancia en la práctica clínica y algunas técnicas relativamente sencillas para su evaluación. Salud Barranquilla 2010;26(1):98-116.

21. Pérez Miguelsanz M, Cabrera Parra W, Varela Moreira $\mathrm{G}$, et al. Distribución regional de la grasa corporal. Uso de técnicas de imagen como herramienta de diagnóstico nutricional. Nutr Hosp 2010;25(2):207-23.

22. Urrejola P, Hernández M, Icaza M, et al. Estimación de masa grasa en niños chilenos: ecuaciones de pliegues subcutáneos vs densitometría de doble fotón. Rev Chil Pediatr 2011;82(6):502-11.

23. Paton J, Beardsmore C, Laverty A, et al. Discrepancies between pediatriclaboratories in pulmonary function results from healthy children. Pediatr Pulmonol 2012;47(6):588-96.

24. Urbankowski T, Przybyłowski T. Methods of airway resistance assessment. Pneumonol Alergol Pol 2016;84(2): 134-41.

25. Lauhkonen E, Koponen P, Nuolivirta K, et al. Obesity and Bronchial Obstruction in Impulse Oscillometry at Age 5-7 Years in a Prospective Post-Bronchiolitis Cohort. Pediatr Pulmonol 2015;50(9):908-14.

26. Rosales R. Antropometría en el diagnóstico de pacientes obesos; una revisión. Nutr Hosp 2012;27(6):1803-9.

27. Godínez Gutiérrez S, Marmolejo Orozco G, Márquez Rodríguez E, et al. La grasa visceral y su importancia en obesidad. Rev Endocrinol Nutr 2002;10(3):121-7.

28. Frainer D, Vasconcelos F, Costa L, et al. Body fat distribution in schoolchildren: a study using the LMS method. Rev Bras Med Esporte 2013;19(5):317-22.

29. McCarthy H, Cole J, Fry T, et al. Body fat reference curves for children. Int J Obes (Lond) 2006;30(4):598-602.

30. Sistema deinformación nacional decalidad delaire. Estación U.C. Maule. Ministerio del Medio Ambiente, Chile. [Acceso: 22 de agosto de 2017]. Disponible en http: / / sinca.mma. gob.cl/index.php/estacion/index/id/205. 